\title{
The monads of classical algebra are seldom weakly cartesian
}

\author{
Maria Manuel Clementino • Dirk Hofmann • \\ George Janelidze
}

Dedicated to Professor H. Inassaridze on the occasion of his eightieth birthday

Received: 5 July 2013 / Accepted: 23 September 2013 / Published online: 13 November 2013

(C) Tbilisi Centre for Mathematical Sciences 2013

\begin{abstract}
This paper begins a systematic study of weakly cartesian properties of monads that determine familiar varieties of universal algebras. While these properties clearly fail to hold for groups, rings, and many other related classical algebraic structures, their analysis becomes non-trivial in the case of semimodules over semirings, to which our main results are devoted. In particular necessary and sufficient conditions on a semiring $\mathrm{S}$, under which the free semimodule monad has: (a) its underlying functor weakly cartesian, (b) its unit a weakly cartesian natural transformation, (c) its multiplication a weakly cartesian natural transformation, are obtained.
\end{abstract}

Communicated by Ronald Brown.

Research partially supported by Centro de Matemática da Universidade de Coimbra (funded by the European Regional Development Fund through the program COMPETE and by the Portuguese Government through the FCT-Fundação para a Ciência e a Tecnologia under the project PEst-C/MAT/UI0324/2011), by Centro de Investigação e Desenvolvimento em Matemática e Aplicações da Universidade de Aveiro/FCT, by the FCT projects MONDRIAN (PTDC/EIA-CCO/108302/2008), and MCANA (PTDC/MAT/120222/2010), and by South African NRF.

M. M. Clementino

Department of Mathematics, CMUC, University of Coimbra, 3001-501 Coimbra, Portugal e-mail:mmc@mat.uc.pt

D. Hofmann

Departamento de Matemática, CIDMA, Universidade de Aveiro, 3810-193 Aveiro, Portugal e-mail: dirk@ua.pt

G. Janelidze ( $\otimes)$

Department of Mathematics and Applied Mathematics, University of Cape Town,

Rondebosch 7701, Cape Town, South Africa

e-mail: George.Janelidze@uct.ac.za 
Keywords Monad - Cartesian - Weakly cartesian - Weak pullback · Variety of algebras $\cdot$ Semiring $\cdot$ Semimodule $\cdot$ Subtractive

Mathematics Subject Classification (1991) $\quad$ 18C15 - 18C20 - 08A62 - 16Y60

\section{Introduction}

There are several areas of applied category theory, from Grothendieck descent theory to the study of higher-dimensional categorical structures, which use so-called cartesian monads and monads satisfying weaker conditions, where the relevant pullbacks are replaced with weak pullbacks. In particular, previous work of the first two authors $[6,7]$ extensively used the fact that the ultrafilter monad on the category Set of sets preserves weak pullbacks and its multiplication is weakly cartesian (that is, satisfying (BC) in the terminology of $[4,5])$.

In this paper we consider several monads on Set that determine familiar varieties of universal algebras, and make an attempt of systematic study of weakly cartesian properties for such monads. Specifically, we are interested if such a monad $T=$ $(T, \eta, \mu)$ on Set has the following properties:

- the functor $T$ preserves weak pullbacks;

- the natural transformations $\eta$ and $\mu$ are weakly cartesian.

(Definition 2.1 below recalls what this means explicitly). The results, including known, new but obvious, and less obvious ones, are:

- Observation 3.1, listing some known varieties determined by cartesian monads.

- Theorem 3.3, which in fact gives wide classes of monads $T=(T, \eta, \mu)$, where $T$ does not preserve weak pullbacks, and where $\mu$ is not weakly cartesian-although we then consider only one specific example.

- Proposition 4.4, which gives another wide class of monads $T$, where the functor $T$ does not preserve weak pullbacks; that class contain monads that determine varieties of groups, rings, modules and all kinds of algebras over rings, and, much more generally, all universal algebras that admit a very weak form of subtraction operation. Some intermediate levels of generality are also mentioned in the same section.

- Theorems 6.4, 7.1, and 8.10, which give necessary and sufficient conditions on a semiring $S$, under which the free $S$-semimodule monad satisfies any of the abovementioned properties. In some sense the case of semimodules is the only case, found so far, where those properties are characterized in terms of non-trivial algebraic conditions rather than just either obviously hold or obviously fail. We therefore devoted the last section to consider semimodules over some particular semirings. Note also that considering varieties of semimodules is the same as considering arbitrary varieties, in which finite coproducts (=free products) canonically coincide with finite products (see e.g. [9]), or, equivalently, pointed varieties with binary biproducts.

The paper is divided, apart from this introduction, into eight sections, whose titles are self-explanatory: 
2. Weakly cartesian monads

3. Some cartesian cases and a related negative result

4. Normal, 0-regular, subtractive, and weakly subtractive varieties

5. A condition that excludes cartesianness

6. Semimodules I: Preliminary remarks and weak cartesianness of the functor $T$

7. Semimodules II: Weak cartesianness for $\eta$

8. Semimodules III: Weak cartesianness of $\mu$ and the main results

9. Semimodules IV: Examples.

\section{Weakly cartesian monads}

Recall that a commutative diagram

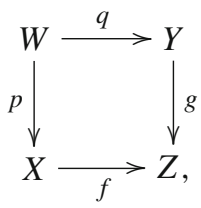

in a category is a weak pullback if for each pair $(u: P \rightarrow X, v: P \rightarrow Y)$ there exists a morphism $t: P \rightarrow W$ (not necessarily unique) such that $p \cdot t=u$ and $q \cdot t=v$. If the category has pullbacks, (2.1) is a weak pullback if and only if the canonical map

$$
\langle p, q\rangle: W \longrightarrow X \times_{Z} Y
$$

is a split epimorphism. Therefore, in the category Set of sets, (2.1) is a weak pullback if and only if $\langle p, q\rangle$ is surjective, that is, for every $x \in X$ and $y \in Y$ with $f(x)=g(y)$, there exists $w \in W$ with $p(w)=x$ and $q(w)=y$.

Definition 2.1 Given a monad $T=(T, \eta, \mu)$ on Set, we say that:

(a) the functor $T$ is weakly cartesian if it preserves weak pullbacks, or, equivalently, transforms pullbacks into weak pullbacks.

(b) the natural transformation $\eta$ is weakly cartesian if, for every map $u: A \rightarrow B$, the naturality square

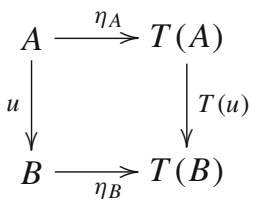

is a weak pullback.

(c) analogously, the natural transformation $\mu$ is weakly cartesian if, for every map $u: A \rightarrow B$, the naturality square 


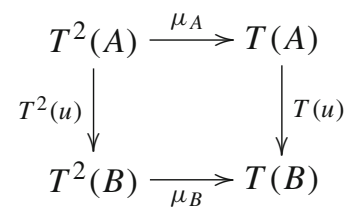

is a weak pullback.

Remarks 2.2 (a) Replacing weak pullbacks with (ordinary) pullbacks in Definitions 2.1, we obtain the conditions that define cartesian monads. Moreover, every cartesian monad obviously satisfies the conditions of Definition 2.1. Monads satisfying these conditions will be called weakly cartesian, as it is done in [18,24,27].

(b) The monads with weakly cartesian functor $T$ (called also condition (BC) on the functor $T$ ) and weakly cartesian $\mu$ (called condition (BC) on the natural transformation $\mu$ ) were considered many times, starting from [4], and including [8]. Weak cartesianness of $\eta$ was not used in those papers since it does not hold for the ultrafilter monad, which was the main example there-except [7], where a special case was used. We will say that the monad $(T, \eta, \mu)$ satisfies $(\mathrm{BC})$ if $T$ and $\mu$ satisfy (BC).

(c) As, e.g., Manes does in his papers [20,21], we call a functor taut if it preserves pullbacks along monomorphisms, while a natural transformation is taut if the naturality squares determined by monomorphisms are pullbacks. Clearly every weakly cartesian functor is taut, and every weakly cartesian natural transformation is taut. A monad $T=(T, \eta, \mu)$ is taut if $T, \eta$ and $\mu$ are taut. We also remark that Möbus introduced this type of monads under the name Alexandrov monad in [22].

Any variety $\mathbf{C}$ of universal algebras is equipped with the free-forgetful adjunction

$$
(F, U, \eta, \varepsilon): \mathbf{C} \longrightarrow \text { Set }
$$

and therefore has its corresponding monad $T=(T, \eta, \mu)$ on Set, in which

$$
T=U F, \eta \text { is as in (2.5), and } \mu=U \varepsilon F \text {. }
$$

We can also write

$$
\operatorname{Set}^{T}=\operatorname{Alg}(\Omega, \Phi),
$$

where $(\Omega, \Phi)$ is any presentation of $\mathbf{C}$ as the category of $\Omega$-algebras satisfying a set $\Phi$ of identities.

In particular, there are two trivial cases:

Example 2.3 Suppose $\Phi$ in (2.7) contains the identity $x=y$, where $x$ and $y$ are distinct variables. Then any $T$-algebra has at most one element, and in particular the same is true for $T(X)$, for any set $X$. The monad $T$ is then called trivial. In fact there are exactly two trivial monads on Set, both of the form (2.6) for some variety $\mathbf{C}$, namely: 
(a) the monad $T$ with $T(X)=1$ (one-element set) for every set $X$-this is the case if and only if the set $\Omega_{0}$ of 0 -ary operators in any (equivalently, in every) presentation (2.7) is non-empty; and

(b) the monad $T$ with

$$
T(X)= \begin{cases}1, & \text { if } X \neq \emptyset \\ \emptyset, & \text { if } X=\emptyset\end{cases}
$$

which is the case if and only if the set $\Omega_{0}$ is empty.

In both cases it is easy to see that $T$ satisfies (BC), but $\eta$ is not weakly cartesian.

From now on we shall always assume that the monad $T$ is non-trivial, or, equivalently, that $\mathbf{C}$ is non-trivial, that is, that $\mathbf{C}$ contains algebras of arbitrarily large cardinality. In particular this implies that every component of $\eta$ is injective.

Remarks 2.4 (a) Since every component of $\eta$ is injective, weak cartesianness of $\eta$ is equivalent to the requirement that every diagram of the form (2.3) is a pullback, that is, it is equivalent to cartesianness of $\eta$. Hence, when $T 1 \cong 1$, weak cartesianness of $\eta$ gives that diagram (2.3) for $u: X \rightarrow 1$ is a pullback, that is, $\eta_{X}$ is an isomorphism for every $X$, and so $T$ is the identity monad.

(b) In contrast to this and Proposition 2.3 of [21] we point out that, if $T$ is non-trivial, $\eta$ is taut. Indeed, consider our diagram (2.3) assuming that $u$ is injective, and suppose $\eta_{B}(b)=T(u)(t)$ for some $b \in B$ and $t \in T(A)$. We shall separately consider the case $b \in u(A)$, and the case $b \notin u(A)$.

Case 1: $b \in u(A)$. We have $b=u(a)$ for some $a \in A$. This gives:

$$
T(u) \eta_{A}(a)=\eta_{B} u(a)=\eta_{B}(b)=T(u)(t)
$$

and so $\eta_{A}(a)=t$, since $T(u)$ is injective.

Case 2: $b \notin u(A)$. Let $C$ be an arbitrary non-empty $T$-algebra, and $c$ and $d$ elements in $C$. Let $f$ and $g$ be any two maps from $B$ to $C$ with $f(b)=c$, $g(b)=d$, and $f(x)=g(x)$ for every $x \in u(A)$, and let $\bar{f}$ and $\bar{g}$ be $T$-algebra homomorphisms $T(B) \rightarrow C$ induced by $f$ and $g$ respectively. We have:

$$
\begin{aligned}
& c=f(b) \quad \text { (by the assumption on } \mathrm{f} \text { ) } \\
& =\bar{f} \eta_{B}(b) \quad \text { (by definition of } \bar{f} \text { ) } \\
& =\bar{f} T(u)(t) \text { (by the assumption on } b \text { and } t) \\
& =\bar{g} T(u)(t) \text { (since } \bar{f} T(u), \bar{g} T(u): T(A) \rightarrow C \text { are } T \text {-algebra } \\
& \text { homomorphisms induced by the maps } f u, g u: A \rightarrow C \\
& \text { respectively, and since these two maps coincide by the } \\
& =d \quad \text { (by similar arguments). }
\end{aligned}
$$

That is, every $T$-algebra has at most one element, making $T$ a trivial monad. This excludes Case 2. 


\section{Some cartesian cases and a related negative result}

Following Carboni and Johnstone [2], Leinster [19] defines a finitary algebraic theory to be strongly regular if it can be presented with identities in which "the same variables appear in the same order, without repetition, on each side". In our notation (2.7) this means that $\Phi$ consists of such identities, and if so, then, motivated by Theorem C.1.1 in [19], we could call C operadic. As we know from [2] (see also [19] and [3]), the corresponding monad is then cartesian, which, among other things, obviously implies the following well-known observation:

Observation 3.1 The monads determining the following varieties are cartesian:

(a) All varieties of the form $\operatorname{Alg}(\Omega, \Phi)$ with $\Phi=\emptyset$. In particular, all varieties in which $\Omega=\Omega_{0}$, that is, each basic operation is 0 -ary, can be presented this way. This includes sets and pointed sets.

(b) All varieties $\mathbf{C}$ of the form $\mathbf{C}=\operatorname{Set}^{M}$, where $M$ is a monoid. In fact, more generally, the same is obviously true for all varieties of the form $\left(C \downarrow\right.$ Set $\left.^{M}\right)$, where $C$ is any $M$-set.

(c) The variety of semigroups.

(f) The variety of monoids.

We should also mention an analogy with Manes' Theorem 4.14 of [20] and Proposition 3.11 of [21], which say that a finitary monad is taut if and only if the corresponding algebraic theory is balanced. Here "balanced", also called "regular" by some other authors, is a notion weaker than what we referred to as "strongly regular": it only requires having the same set of variables on each side. This fact is very important for us since all easy consequences of our results involving taut monads can also be obtained from it.

In spite of Observation 3.1(b), there are varieties of $M$-sets that are not even weakly cartesian. This follows from Theorem 3.3 below, whose formulation and proof require to adjust the universal-algebraic and the monad-theoretic terminology and notation. For our purposes it actually suffices to mention that:

- The universal-algebraic expression $t=t(x)$, for a unary term $t$, simply means that $t$ belongs to $T(\{x\})$. And if $t$ belongs to $T(\{x\}),\left(A, h_{A}\right)$ is a $T$-algebra, and $a$ is an element in $A$, then $t(a)=h_{A} T(\underline{a})(t)$ is the image of $t$ under the $T$-algebra homomorphism $\left(T(\{x\}), \mu_{\{x\}}\right) \rightarrow\left(A, h_{A}\right)$, induced by the map $\underline{a}:\{x\} \rightarrow A$ sending $x$ to $a$. In particular, if $t^{\prime} \in T(\{x\})$ is another such unary term, then $t\left(t^{\prime}\right)=$ $\mu_{\{x\}} T\left(t^{\prime}\right)(t)$, that is, $t\left(t^{\prime}\right)$ is the image of $t$ under the $T$-algebra homomorphism $\left(T(\{x\}), \mu_{\{x\}}\right) \rightarrow\left(T(\{x\}), \mu_{\{x\}}\right)$, induced by the map $\underline{t}^{\prime}:\{x\} \rightarrow T(\{x\})$ sending $x$ to $t^{\prime}$. Furthermore, $t^{\prime}=\eta_{\{x\}}(x)$ makes $\mu_{\{x\}} T\left(t^{\prime}\right)(t)=\mu_{\{x\}} T\left(\eta_{\{x\}}\right)(t)=t$, and so $t=t\left(\eta_{\{x\}}(x)\right)$; this nicely agrees with the universal-algebraic expression $t=t(x)$ up to identifying $x$ with its image in $T(\{x\})$.

- In the notation above, if $u: A \rightarrow B$ is a $T$-algebra homomorphism, then

$$
u(t(a))=t(u(a))
$$

Indeed, $u(t(a))=u h_{A} T(\underline{a})(t)=h_{B} T(u) T(\underline{a})(t)=h_{B} T(u(\underline{a}))(t)=$ $h_{B} T(u(a))(t)=t(u(a))$. 
- When $A$ is equipped with a $T$-algebra structure $h_{A}$, the (well-defined) element $t(a)$ of $A$ should not be confused with the element $t\left(\eta_{A}(a)\right)$ of $T(A)$. These elements are only related by

$$
t(a)=h_{A}\left(t\left(\eta_{A}(a)\right)\right) .
$$

since

$$
t\left(\eta_{A}(a)\right)=T(\underline{a})(t),
$$

which is easy to check:

$$
t\left(\eta_{A}(a)\right)=\mu_{A} T\left(\underline{\eta_{A}(a)}\right)(t)=\mu_{A} T\left(\eta_{A} \underline{a}\right)(t)=\mu_{A} T\left(\eta_{A}\right) T(\underline{a})(t)=T(\underline{a})(t) .
$$

- By a pseudo-constant unary term we mean a term $t \in T(\{x\})$ such that, for every $T$-algebra $A$ and every two elements $a$ and $a^{\prime}$ in $A$, we have $t(a)=t\left(a^{\prime}\right)$ in $A$. In the universal-algebraic terminology, a unary term $t$ is pseudo-constant if and only if, for some distinct variables $x$ and $y$, the identity $t(x)=t(y)$ holds in C. Note that whenever $T(\emptyset)$ is non-empty, or, equivalently, $\Omega_{0}$ is nonempty, every pseudoconstant term $t \in T(\{x\})$ is constant, that is, it belongs to the image of $T(\emptyset)$ in $T(\{x\})$. The notion of pseudo-constant term has an obvious non-unary version of course.

The following lemma would be considered trivial in universal algebra; nevertheless we shall prove it since it plays an important role in the proof of Theorem 3.3(b) below.

Lemma 3.2 Let $S$ be a set and $s$ and $s^{\prime}$ elements in $S$. If $s \neq s^{\prime}$ and $t$ is not a pseudo-constant term, then $t\left(\eta_{A}(s)\right) \neq t\left(\eta_{A}\left(s^{\prime}\right)\right)$.

Proof Let $\left(A, h_{A}\right)$ be a $T$-algebra and $a$ and $a^{\prime}$ elements in $A$. Let $f: S \rightarrow A$ be any map with $f(s)=a$ and $f\left(s^{\prime}\right)=a^{\prime}$, and $g:\left(T(S), \mu_{S}\right) \rightarrow\left(A, h_{A}\right)$ be a $T$-algebra homomorphism such that $g \eta_{S}=f$; the existence of such $g$ follows from the fact that $\eta_{S}$ makes $\left(T(S), \mu_{S}\right)$ the free $T$-algebra on $S$. Using (3.1) (for $g$ playing the role of $u)$, we obtain

$$
t(a)=t(f(s))=t\left(g\left(\eta_{S}(s)\right)\right)=g\left(t\left(\eta_{A}(s)\right)\right),
$$

and similarly $t\left(a^{\prime}\right)=g\left(t\left(\eta_{A}\left(s^{\prime}\right)\right)\right)$. Therefore the equality $t\left(\eta_{A}(s)\right)=t\left(\eta_{A}\left(s^{\prime}\right)\right)$ would imply that $t$ is a pseudo-constant term.

Theorem 3.3 (a) If $\mathbf{C}$ admits a pseudo-constant term that is not constant, then the functor $T$ is not weakly cartesian (and not even taut).

(b) Let $\mathbf{C}=\operatorname{Set}^{T}=\operatorname{Alg}(\Omega, \Phi)$ be a variety of universal algebras that admits nonpseudo-constant unary terms $t, t^{\prime} \in T(\{x\})$, for which $t\left(t^{\prime}\right)$ is a pseudo-constant term. Then the natural transformation $\mu$ is not weakly cartesian (and not even taut). 
Proof (a): Let $t \in T(\{x\})$ be a pseudo-constant non-constant term, and let us choose in the pullback diagram (2.1): $X=\{x\}, Y=\{y\}, Z=\{x, y\}$, with $f$ and $g$ the inclusion maps. Then $\langle T(p), T(q)\rangle: T(W) \rightarrow T(X) \times_{T(Z)} T(Y)$ cannot be surjective since:

$-T(W)=T(\emptyset)$ is empty - otherwise there would be no pseudo-constant nonconstant terms;

- $T(X) \times_{T(Z)} T(Y)$ is non-empty since it contains the element determined by the pair $\left(t\left(\eta_{X}(x)\right), t\left(\eta_{Y}(y)\right)\right)$.

(b): Let $u: A \rightarrow B$ by any map of sets with non-empty $A$ and the cardinality of $B$ strictly larger than the cardinality of $T^{2}(A)$, and $a$ any fixed element in $A$. For each $b \in B$, using (3.2), then (3.3), we obtain

$$
\begin{aligned}
\mu_{B}\left(t\left(\eta_{T(B)}\left(t^{\prime}\left(\eta_{B}(b)\right)\right)\right)\right) & =t\left(t^{\prime}\left(\eta_{B}(b)\right)\right) & & (\text { by }(3.2)) \\
& =t\left(T(\underline{b})\left(t^{\prime}\right)\right) & & (\text { by }(3.3)) \\
& =T(\underline{b})\left(t\left(t^{\prime}\right)\right) & & (\text { by }(3.1)) \\
& =\left(t\left(t^{\prime}\right)\right)\left(\eta_{B}(b)\right) & & \text { by }(3.3)) \\
& =\left(t\left(t^{\prime}\right)\right)\left(T(u)\left(\eta_{A}(a)\right)\right) & & \text { (since } t\left(t^{\prime}\right) \text { is a pseudo-constant term) }
\end{aligned}
$$

Therefore the set $P_{a}=\left\{\left(\left(t\left(\eta_{T(B)}\left(t^{\prime}\left(\eta_{B}(b)\right)\right)\right), T(u)\left(\eta_{A}(a)\right)\right) \mid b \in B\right\}\right.$ can be considered as a subset in the pullback $T^{2}(B) \times_{T(B)} T(A)$. After that, since the cardinality of $B$ is strictly larger than the cardinality of $T^{2}(A)$, it suffices to show that, for $b, b^{\prime} \in B$, we have

$$
b \neq b^{\prime} \Rightarrow t\left(\eta_{T(B)}\left(t^{\prime}\left(\eta_{B}(b)\right)\right)\right) \neq t\left(\eta_{T(B)}\left(t^{\prime}\left(\eta_{B}\left(b^{\prime}\right)\right)\right)\right) .
$$

But we have

$b \neq b^{\prime} \Rightarrow t^{\prime}\left(\eta_{B}(b)\right) \neq t^{\prime}\left(\eta_{B}\left(b^{\prime}\right)\right) \Rightarrow t\left(\eta_{T(B)}\left(t^{\prime}\left(\eta_{B}(b)\right)\right)\right) \neq t\left(\eta_{T(B)}\left(t^{\prime}\left(\eta_{B}\left(b^{\prime}\right)\right)\right)\right)$,

where both implications follow from Lemma 3.2 since $t^{\prime}$ and $t$ are not pseudo-constant terms.

The simplest example seems to be:

Example 3.4 Let $\mathbb{N}$ be the additive monoid of natural numbers, and $\mathbf{C}$ the variety of $\mathbb{N}$-sets determined by the identity $2 x=2 y$. Then the corresponding monad $T=$ $(T, \eta, \mu)$ can be described as follows:

$-T(\emptyset)=\emptyset$;

- If $X$ is a non-empty set, then $T(X)$ is the disjoint union of $\{0,1\} \times X$ with a one-element set, say $\{\infty\}$;

$-\eta_{X}: X \rightarrow T(X)$ is defined by $\eta_{X}(x)=(0, x)$;

$-\mu_{X}: T^{2}(X) \rightarrow T(X)$ is defined by

$$
\begin{aligned}
\mu_{X}(k,(l, x)) & = \begin{cases}(k+l, x), & \text { if } k+l \leq 1, \\
\infty, & \text { if } k+l=2,\end{cases} \\
\mu_{X}(k, \infty) & =\mu_{X}(\infty)=\infty
\end{aligned}
$$


This monad has a pseudo-constant non-constant term, namely $\infty$, and so $T$ is not weakly cartesian by Theorem 3.3(a). Furthermore, for this monad $t=(1, x) \in T(\{x\})$ is not pseudo-constant term, while $t(t)=\infty$ is a pseudo-constant term. Therefore, by Theorem 3.3(b), $\mu$ is not weakly cartesian. It is easy to check, however, that $\eta$ is.

Remark 3.5 It is easy to check that modifying Example 3.4 by only adding an arbitrary (non-zero) number of constants and still requiring only the identity $2 x=2 y$, and possibly identities involving only constants, we obtain a monad with $T$ and $\eta$ weakly cartesian, but, still by Theorem 3.3(b), $\mu$ is not weakly cartesian.

\section{Normal, 0-regular, subtractive, and weakly subtractive varieties}

The following conditions on a variety $\mathbf{C}$ of universal algebras are well known to be equivalent:

- $\mathbf{C}$ is pointed as a category, that is, each hom-set $\operatorname{hom}_{C}(A, B)$ has a distinguished element $0_{A, B}$, such that, for every morphism $\alpha: A^{\prime} \rightarrow A$ and every morphism $\beta: B \rightarrow B^{\prime}$, we have $\beta 0_{A, B} \alpha=0_{A^{\prime}, B^{\prime}}$;

- the free algebra in $\mathbf{C}$ on the empty set (=the initial object in $\mathbf{C}$ ) has exactly one element ( $=$ is isomorphic to the terminal object in $\mathbf{C}$ );

- the set $\Omega_{0}$ of 0 -ary operators in $\mathbf{C}$ is non-empty, and every two constant terms are equal to each other in each object in $\mathbf{C}$;

- every algebra in $\mathbf{C}$ has a one-element subalgebra;

- every algebra in $\mathbf{C}$ has a unique one-element subalgebra, and, for every morphism $\alpha: A^{\prime} \rightarrow A$, that subalgebra is the image of $0_{A^{\prime}, A}$.

When these conditions hold, we shall simply say that $\mathbf{C}$ is pointed, and we shall use the symbol 0 for

- each of the morphisms $0_{A, B}$;

- the initial object in $\mathbf{C}$;

- the unique subalgebra in any algebra in $\mathbf{C}$;

- the unique element in that subalgebra;

we will also say that 0 is the unique constant $(=0$-ary basic operation) in $\mathbf{C}$.

When $\mathbf{C}$ is pointed, every morphism $u: A \rightarrow B$ has a kernel

$$
\operatorname{ker}(u): \operatorname{Ker}(u) \longrightarrow A,
$$

which can be described, up to isomorphism, as the inclusion map of $\{a \in A \mid u(a)=0\}$ into $A$, and a cokernel

$$
\operatorname{coker}(u): B \longrightarrow \operatorname{Coker}(u),
$$

which can be described, up to an isomorphism, as the canonical morphism from $B$ to its quotient by the smallest congruence $\sim$, under which $u(a) \sim 0$ for each $a \in A$. The surjective homomorphisms (=regular epimorphisms) that occur as cokernels are called normal epimorphisms. Furthermore, following [16], we shall say that $\mathbf{C}$ is 
normal if every regular epimorphism in $\mathbf{C}$ is a normal epimorphism. It is well known that every pointed BIT variety in the sense of [25] (=ideal determined varieties in the sense of [11]) is normal. In particular this includes all semi-abelian varieties (see [13], although in the case of pointed varieties semi-abelianness is equivalent to Bourn protomodularity [1]), and (therefore, although known independently) all varieties of groups with multiple operators in the sense of [12], and so many varieties of classical algebra, e.g. of groups, non-unital rings, modules and various kinds of non-unital algebras over rings, etc. It also includes, say, Heyting semilattices (see e.g. [17]; note, however, that the role of 0 there is played by the constant 1), but not, for example, semilattices, lattices, monoids, etc.

Slightly more generally, instead of normal varieties we could consider what universal algebraists call 0-regular varieties (see [14-16] for explanations and references). Those are not necessarily pointed, but have a distinguished constant 0 such that every congruence on any given algebra is completely determined by its 0 -class. An obvious reason to consider the non-pointed case is to include important examples, such as unital rings and unital algebras over rings, Boolean and Heyting algebras, etc.

Another class of varieties containing the above-mentioned varieties of classical algebra is the class of subtractive varieties in the sense of Ursini [26] (in fact appeared already in [11]). They are defined as varieties having a distinguished constant 0 and a binary term $s$ with the identities $s(x, x)=0$ and $s(0, x)=x$ (among others). As shown in [11], a (not necessarily pointed) variety is ideal determined if and only if it is 0 -regular and subtractive at the same time, with the same 0 . Writing briefly,

$$
\text { ideal determined }=0 \text {-regular } \cap \text { subtractive, }
$$

and we are going to introduce weakly subtractive varieties with

$$
\text { weakly subtractive } \supset \text { 0-regular } \cup \text { subtractive, }
$$

as follows:

Definition 4.1 A variety $\mathbf{C}$ is said to be weakly subtractive if it admits a constant 0 and a non-pseudo-constant $n$-ary term $s$ satisfying the identity $s(x, \ldots, x)=0$ and not satisfying the identity $s\left(x_{1}, \ldots, x_{n}\right)=0$.

Observation 4.2 (a) Every subtractive variety is weakly subtractive indeed, as trivially follows from the definitions.

(b) Every 0-regular variety is also weakly subtractive as follows, e.g., from Corollary 1.7 in [11].

(c) For the subtractive and 0-regular varieties the desired term $s$ there is actually binary. On the other hand, in Definition 4.1, the cases $n=0$ and $n=1$ are automatically excluded, since in those cases our requirements on $s$ become contradictory.

For a morphism $u: A \rightarrow B$ in a variety $\mathbf{C}$ having a distinguished constant 0 , we shall still write $\operatorname{Ker}(u)=\{a \in A \mid u(a)=0\}$, even though this $\operatorname{Ker}(u)$ does not have to be a subalgebra in $A$ in general. The following simple observation characterizes weak subtractivity of $\mathbf{C}$ in terms of the corresponding monad on Set: 
Observation 4.3 Let $\mathrm{C}$ be a variety having a distinguished constant 0 and $T=$ $(T, \eta, \mu)$ be the corresponding monad on Set. The following conditions are equivalent:

(i) $\mathbf{C}$ is weakly subtractive;

(ii) there exists a map $f: X \rightarrow Y$ with $\operatorname{Ker}(T(f)) \neq 0$;

(iii) for each cardinal number $\mathfrak{m}$ there exists a map $f: X \rightarrow Y$ with $\operatorname{Ker}(T(f)$ ) having cardinality $\geq \mathfrak{m}$.

Let us only explain the implication (ii) $\Rightarrow$ (iii), which might seem less obvious. Condition (ii) means that the variety $\mathbf{C}$ admits a, say $n$-ary, term $t$ with $t\left(x_{1}, \ldots, x_{n}\right) \neq$ 0 in $T(X)$ and $t\left(f\left(x_{1}\right), \ldots, f\left(x_{n}\right)\right)=0$ in $Y$, for some $f: X \rightarrow Y$, and some elements $x_{1}, \ldots, x_{n}$ in $X$. Let us use:

- a set $S$ of cardinality $\geq \mathfrak{m}$;

- the map $S \times f: S \times X \rightarrow S \times Y$ defined by $(S \times f)(s, x)=(s, f(x))$.

We have:

(a) $t(0, \ldots, 0)=0$ in any $T$-algebra $A$. Indeed, since $T(Y)$ is the free $T$-algebra on $Y$, the map $u: Y \rightarrow T(A)$, sending all elements of $Y$ to 0 , extends to a homomorphism $v: T(Y) \rightarrow T(A)$, and we have

$$
\begin{aligned}
t(0, \ldots, 0) & =t\left(u f\left(x_{1}\right), \ldots, u f\left(x_{n}\right)\right)=t\left(v f\left(x_{1}\right), \ldots, v f\left(x_{n}\right)\right) \\
& =v\left(t\left(f\left(x_{1}\right), \ldots, f\left(x_{n}\right)\right)\right)=v(0)=0 .
\end{aligned}
$$

(b) $t\left((S \times f)\left(s, x_{1}\right), \ldots,(S \times f)\left(s, x_{n}\right)\right)=0$ in $T(S \times X)$ for every $s$ in $S$. Indeed, let $w: Y \rightarrow S \times Y$ be defined by $w(y)=(s, y)$-then

$$
\begin{aligned}
t\left((S \times f)\left(s, x_{1}\right), \ldots,(S \times f)\left(s, x_{n}\right)\right) & =T(w)\left(t\left(f\left(x_{1}\right), \ldots, f\left(x_{n}\right)\right)\right) \\
& =T(w)(0)=0,
\end{aligned}
$$

since $T(w)$ is a homomorphism of algebras.

(c) As follows from (b), $t\left(\left(s, x_{1}\right), \ldots,\left(s, x_{n}\right)\right)$ is in $\operatorname{Ker}(T(S \times f))$ for each $s$ in $S$, after which it suffices to show that

$$
s \neq s^{\prime} \Longrightarrow t\left(\left(s, x_{1}\right), \ldots,\left(s, x_{n}\right)\right) \neq t\left(\left(s^{\prime}, x_{1}\right), \ldots,\left(s^{\prime}, x_{n}\right)\right)
$$

For, given $s \neq s^{\prime}$ in $S$, consider any map $g: S \times X \rightarrow X$ with $g(s, x)=x$ and $g\left(s^{\prime}, x\right)=0$, for each $x$ in $X$. We have

$$
\begin{aligned}
& T(g)\left(t\left(\left(s, x_{1}\right), \ldots,\left(s, x_{n}\right)\right)\right)=t\left(x_{1}, \ldots, x_{n}\right) \neq 0, \text { and } \\
& T(g)\left(t\left(\left(s^{\prime}, x_{1}\right), \ldots,\left(s^{\prime}, x_{n}\right)\right)\right)=t(0, \ldots, 0)=0 \text { by (a). }
\end{aligned}
$$

Therefore $t\left(\left(s, x_{1}\right), \ldots,\left(s, x_{n}\right)\right) \neq t\left(\left(s^{\prime}, x_{1}\right), \ldots,\left(s^{\prime}, x_{n}\right)\right)$, as desired.

And this observation helps to prove:

Proposition 4.4 If $\mathbf{C}$ is weakly subtractive, then $T$ is not a weakly cartesian functor (not even taut). 
Proof Let $f: X \rightarrow Y$ be as in 4.3(iii) with $\mathfrak{m}$ larger than the cardinality of $T(\emptyset)$. Consider the pullback

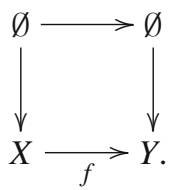

The resulting canonical map $T(\emptyset) \rightarrow T(X) \times_{T(Y)} T(\emptyset)$ cannot be surjective since $T(X) \times_{T(Y)} T(\emptyset)$ contains the set $\operatorname{Ker}(T(f)) \times\{0\}$, whose cardinality is larger than the cardinality of $T(\emptyset)$.

\section{A condition that excludes cartesianness}

Unlike Sect. 2, due to the simplicity of the data considered in this section, we shall freely use the universal-algebraic expressions $t=t(x), t(y)$ instead of $t\left(\eta_{Y}(y)\right)$, etc. The section is devoted to a simple result showing that many familiar monads are not cartesian:

Proposition 5.1 Suppose C admits a commutative binary operation which is not pseudo-constant, that is, admits a binary term $t$ satisfying $t(x, y)=t(y, x)$ and not $t\left(x, x^{\prime}\right)=t\left(y, y^{\prime}\right)$, where $x, x^{\prime}, y, y^{\prime}$ are pairwise distinct variables. Then the functor $T$ does not preserve pullbacks.

Proof Let us choose the pullback (2.1) using pairwise distinct elements $x, x^{\prime}, y, y^{\prime}$ as follows:

- $X$ is any set containing $x$ and $x^{\prime}$;

$-Y$ is any set containing $y$ and $y^{\prime}$;

- $Z$ is a one-element set (which uniquely determines $f$ and $g$ ).

Then $W=X \times Y$, and so $T(W)$ contains the elements $t\left((x, y),\left(x^{\prime}, y^{\prime}\right)\right)$ and $t\left(\left(x, y^{\prime}\right),\left(x^{\prime}, y\right)\right)$; moreover $t\left((x, y),\left(x^{\prime}, y^{\prime}\right)\right) \neq t\left(\left(x, y^{\prime}\right),\left(x^{\prime}, y\right)\right)$ since the elements $(x, y),\left(x^{\prime}, y^{\prime}\right),\left(x, y^{\prime}\right),\left(x^{\prime}, y\right)$ are pairwise distinct. On the other hand,

$$
\begin{aligned}
\langle T(p), T(q)\rangle\left(t\left((x, y),\left(x^{\prime}, y^{\prime}\right)\right)\right) & =\left(t\left(x, x^{\prime}\right), t\left(y, y^{\prime}\right)\right)=\left(t\left(x, x^{\prime}\right), t\left(y^{\prime}, y\right)\right) \\
& =\langle T(p), T(q)\rangle\left(t\left(\left(x, y^{\prime}\right),\left(x^{\prime}, y\right)\right)\right) .
\end{aligned}
$$

That is, $\langle T(p), T(q)\rangle$ is not injective.

Marek Zawadowski told us that this proposition also follows from results of [23].

\section{Semimodules I: preliminary remarks and weak cartesianness of the functor $T$}

From now on, C will denote the variety $S$-SMod of semimodules over a semiring $S$. This and the next two sections will be devoted to the analysis of weak cartesianness of the functor $T$ and of the natural transformations $\eta, \mu$, respectively, for the corresponding (free $S$-semimodule) $\operatorname{monad} T=(T, \eta, \mu)$. 
Let us recall that an $S$-semimodule is a commutative monoid $A$, written additively, and equipped with a scalar multiplication $S \times A \rightarrow A$, written as $(s, a) \mapsto s a$, and satisfying the identities

$1 a=a, s(t a)=(s t) a, 0 a=0,(s+t) a=s a+t a, s 0=0, s(a+b)=s a+s b$,

in obvious notation. Let us also mention that $S$-SMod is enriched in the category of commutative monoids, and its biproducts

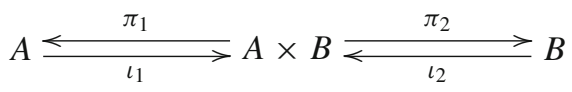

are the usual cartesian product $A \times B$ with the product projections $\pi_{1}$ and $\pi_{2}$, and $\iota_{1}$ and $\iota_{2}$ defined by $\iota_{1}(a)=(a, 0)$ and $\iota_{2}(b)=(0, b)$ respectively. This coincidence of binary products and coproducts implies the preservation of arbitrary coproducts by the functor $T$, and, either using this fact or directly, the monad $T=(T, \eta, \mu)$ can be described as follows:

- for any set $X, T(X)$ is the set of all maps $t: X \rightarrow S$ for which the set $\{x \in$ $X \mid t(x) \neq 0\}$ is finite;

- for a map $f: X \rightarrow Y$, we have $T(f)(t)(y)=\sum_{f(x)=y} t(x)$;

$-\eta_{X}: X \rightarrow T(X)$ is the map that has $\eta_{X}(x)(x)=1$ and $\eta_{X}(x)(y)=0$ for $x \neq y$;

$-\mu_{X}: T^{2}(X) \rightarrow T(X)$ is the map defined by $\mu_{X}(u)(x)=\sum_{t \in T(X)} u(t) t(x)$, where all possibly infinite sums make sense since they involve only finitely many non-zero summands.

We will analyze weak cartesianness of the functor $T$ in several steps.

Lemma 6.1 The following conditions are equivalent:

(i) the T-image of (2.1) is a weak pullback whenever $Y=\emptyset$;

(ii) the $T$-image of (2.1) is a weak pullback whenever the numbers of elements in $X$, $Y$, and $Z$ are 2,0 , and 1 respectively;

(iii) no non-zero element in $S$ has an additive inverse.

Proof If $Y=\emptyset$, then $W=\emptyset$ and $T(W)=T(Y)=\{0\}$, and so the $T$-image of (2.1) is a weak pullback if and only if the kernel of $T(f): T(X) \rightarrow T(Z)$ is $\{0\}$. And when $X$ and $Z$ are as in (ii), the map $T(f): T(X) \rightarrow T(Z)$ can be identified with the addition map $+: S \times S \rightarrow S$, which makes the equivalence (ii) $\Leftrightarrow$ (iii) obvious. Since the implication (i) $\Rightarrow$ (ii) is trivial, it only remains to prove the implication (iii) $\Rightarrow$ (i).

Suppose $\alpha: X \rightarrow S$ belongs to the kernel of $T(f)$. Then, for every $z$ in $Z$, we have $\sum_{f(x)=z} \alpha(x)=0$, and, as follows from (iii), this means that $\alpha(x)=0$ for every $x$ in $X$. That is, $\alpha=0$ in $T(X)$. 
Lemma 6.2 For the weak pullback diagrams
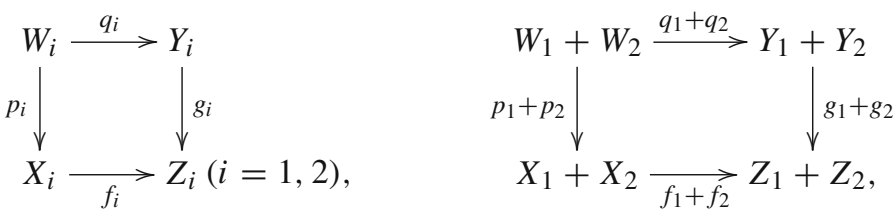

where + denotes the coproduct (=disjoint union) of sets, if $T$ preserves the left-hand weak pullbacks, then it also preserves the right-hand weak pullback.

Proof Just observe that the free $S$-semimodule functor preserves coproducts and that finite coproducts of $S$-semimodules are canonically isomorphic to products.

Lemma 6.3 The following conditions are equivalent:

(i) the T-image of (2.1) is a weak pullback whenever $f$ and $g$ are surjective;

(ii) the T-image of (2.1) is a weak pullback whenever the numbers of elements in $X$, $Y$ and $Z$ are 2, 2 and 1 respectively.

(iii) for every $a, b, c, d$ in $S$ with $a+b=c+d$, there exist $x, y, z, t$ in $S$ with

$$
x+y=a, z+t=b, x+z=c, y+t=d .
$$

(iv) for every natural $m$ and $n$, and $s_{1}, \ldots, s_{m}$ and $t_{1}, \ldots, t_{n}$ in $S$ with $s_{1}+\cdots+s_{m}=$ $t_{1}+\cdots+t_{n}$, there exists $a\{1, \ldots, m\} \times\{1, \ldots, n\}$-indexed family $\left(x_{i j}\right)$ of elements in $S$ with

$$
\begin{gathered}
x_{i 1}+\cdots+x_{i n}=s_{i} \quad \text { for all } i=1, \ldots, m \text { and } \\
x_{1 j}+\cdots+x_{m j}=t_{j} \quad \text { for all } j=1, \ldots, n .
\end{gathered}
$$

Proof When $X, Y$, and $Z$ are as in (ii), the $T$-image of (2.1) can be presented as

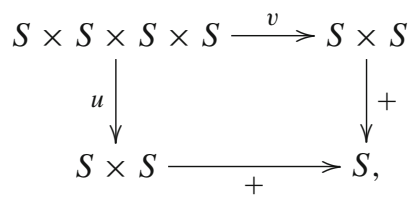

where $u$ and $v$ are defined by $u(x, y, z, t)=(x+y, z+t)$ and $v(x, y, z, t)=$ $(x+z, y+t)$ respectively, which makes the equivalence (ii) $\Leftrightarrow$ (iii) obvious. Since the implication (i) $\Rightarrow$ (ii) is trivial, it only remains to prove the implications (iii) $\Rightarrow$ (iv) and (iv) $\Rightarrow$ (i).

(iii) $\Rightarrow$ (iv) is an elementary exercise. We can use induction in $m$ and in $n$, and since $m$ and $n$ play "symmetric roles", we can assume that there exist an $\{1, \ldots, m\} \times$ $\{2, \ldots, n\}$-indexed family $\left(y_{i j}\right)$ of elements in $S$ with

$$
\begin{gathered}
y_{i 2}+\cdots+y_{i n}=s_{i} \quad \text { for all } i=1, \ldots, m, \text { and } \\
y_{1 j}+\cdots+y_{m j}=u_{j} \quad \text { for all } j=2, \ldots, n,
\end{gathered}
$$


where $u_{2}=t_{1}+t_{2}$ and $u_{j}=t_{j}$ for $j=3, \ldots, n$. After that we can take $x_{i j}=y_{i j}$ for $j=3, \ldots, n$, and define $x_{i j}$ for $j=1,2$ in such a way that

$x_{i 1}+x_{i 2}=y_{i 2} \quad$ for all $i=1, \ldots, m$, and $x_{1 j}+\cdots+x_{m j}=t_{j} \quad$ for all $j=1,2$.

This, using induction in $m$, reduces to (iii).

(iv) $\Rightarrow$ (i): Since the functor $T$ preserves filtered colimits, it suffices to consider the case of finite $X, Y$, and $Z$. Moreover, thanks to Lemma 6.2, we can assume that $Z$ is a one-element set. But assuming $Z$ in (i) to be a one-element set (and $X \neq \emptyset \neq Y$ ) makes (i) obviously equivalent to (iv), just like (ii) is equivalent to (iii).

Theorem 6.4 The following conditions are equivalent:

(i) $T$, as a functor, is weakly cartesian;

(ii) S satisfies the conditions of Lemmas 6.1 and 6.3 .

Proof (i) $\Rightarrow$ (ii) follows from Lemmas 6.1 and 6.3.

(ii) $\Rightarrow$ (i): Assuming that conditions 6.1(iii) and 6.3(iii) hold, we have to show that $T$-images of arbitrary pullbacks (2.1) are weak pullbacks. Using filtered colimits again this reduces to the case of pullbacks of finite sets, and then, using Lemma 6.2, we can assume that $Z$ in (2.1) is a one-element set. After this we just apply Lemmas 6.1 and 6.3 .

Remark 6.5 Using similar arguments it is easy to show that the functor $T$ is taut if and only if no non-zero element in $S$ has an additive inverse.

\section{Semimodules II: weak cartesianness for $\eta$}

Theorem 7.1 The following conditions are equivalent:

(i) $\eta$ is weakly cartesian;

(ii) If $s+s^{\prime}=1$ in $S$, then either $s=0$, or $s^{\prime}=0$. In particular no non-zero element of $S$ has an additive inverse.

Proof (i) $\Rightarrow$ (ii): When $A$ is a two-element set, say, $A=\left\{a, a^{\prime}\right\}$, and $B$ is a one-element set, say, $B=\{b\}$, diagram (2.3) becomes

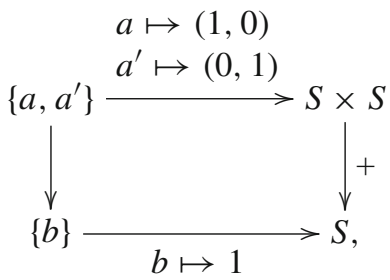

and, obviously, diagram (7.1) is a (weak) pullback if and only if condition (ii) holds. 
(ii) $\Rightarrow$ (i): Suppose, in diagram (2.3), we have $\eta_{B}(b)=T(u)(t)$ for some $b \in B$ and some $t: A \rightarrow S$ in $T(A)$. Then we have:

$$
\sum_{u(a)=b} t(a)=1 \text { and } \sum_{u(a)=b^{\prime}} t(a)=0 \quad \text { for } b^{\prime} \neq b \text { in } B .
$$

But, under condition (ii), (7.2) is equivalent to the existence of (a unique) $a \in A$ with

$$
t(a)=1 \text { and } \forall x \in A \quad(x \neq a \Rightarrow t(a)=0),
$$

and (7.3) implies that the image of $a$ under the canonical map $A \rightarrow T(A) \times_{T(B)} B$ is the element determined by the pair $(b, t)$. This proves (i).

\section{Semimodules III: weak cartesianness of $\mu$ and the main results}

For $S$ and $T=(T, \eta, \mu)$ as in the two previous sections, the analysis of weak cartesianness for $\mu$, which this section is devoted to, is rather complicated and we will use weak cartesianness for the functor $T$ in it. The first of our auxiliary results is obvious and surely known to many readers:

\section{Lemma 8.1 Let}

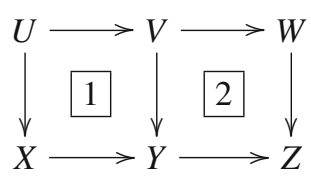

be a commutative diagram in the category of sets. Then:

(a) if 1 and 2 are weak pullbacks, then so is the rectangle 112 ;

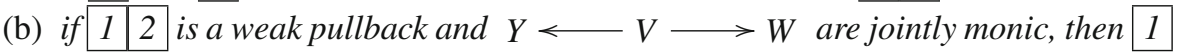
is a weak pullback;

(c) if 1 2 2 is a weak pullback and the map $X \rightarrow Y$ is surjective, then 2 is a weak pullback.

Furthermore:

(d) the class of weak pullback diagrams of sets is closed under products in the category of all square diagrams in the category of sets;

(e) for any weak pullback diagram (2.1) and any map $h: X \rightarrow E$, the diagram

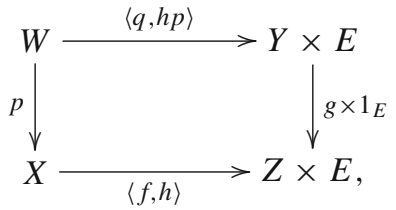

is a weak pullback. 
Lemma 8.2 The class $\mathcal{U}$ of all maps $u: A \rightarrow B$ for which diagram (2.4) is a weak pullback has the following properties:

(a) it contains all bijections and is closed under composition and filtered colimits;

(b) if $T$ is a weakly cartesian functor, then $\mathcal{U}$ is closed under coproducts with bijections.

Proof (a): The fact that $\mathcal{U}$ contains all bijections is obvious. Closedness under composition easily follows from Lemma 8.1(a). Closedness under filtered colimits follows from the fact that the functor $T$ preserves filtered colimits.

(b): We have to prove that, if the functor $T$ is weakly cartesian and diagram (2.4) is a weak pullback, then, for any set $E$, the diagram

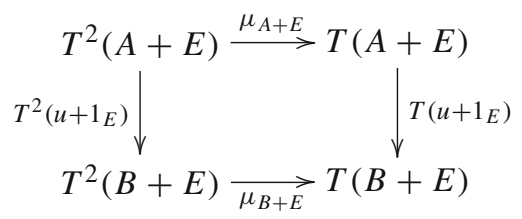

also is a weak pullback; here + stands for coproducts in the category of sets, that is, for disjoints unions (and induced maps between disjoint unions). For that, consider the commutative diagram

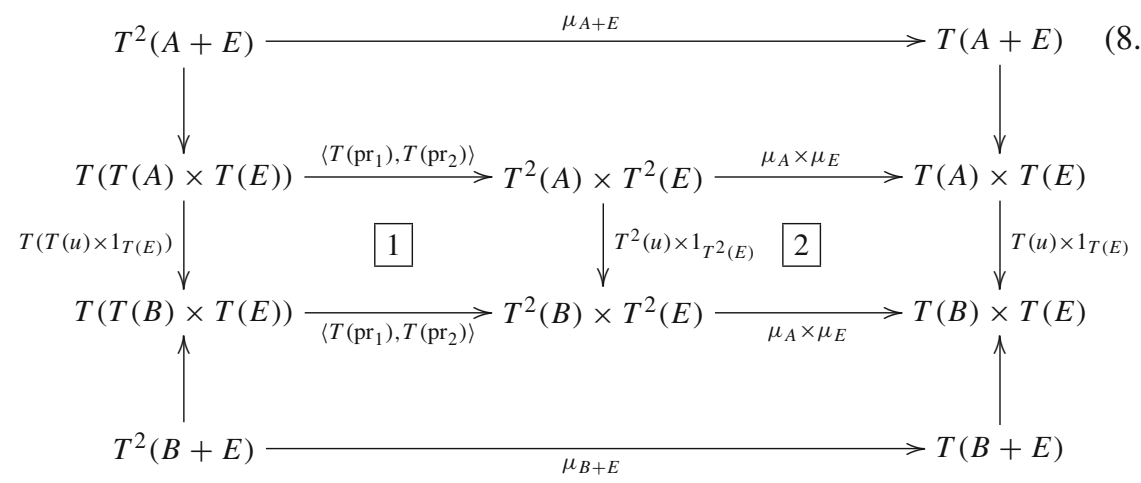

in which the unlabeled arrows are canonical bijections, and observe:

- the square 1 is a weak pullback, which follows from weak cartesianness of the functor $T$ and Lemma 8.1(e);

- the square 2 is a weak pullback, which follows from the fact that so is diagram (2.4) and Lemma 8.1(d);

- therefore 1 2 2 is a weak pullback by Lemma 8.1(a);

- Since the two top and two bottom vertical arrows in (8.4) are bijections, and 102 is a weak pullback, diagram (8.3) also is a weak pullback.

Corollary 8.3 If $T$ is a weakly cartesian functor, then the natural transformation $\mu$ is weakly cartesian if diagram (2.4) is a weak pullback whenever A is either empty or is a two-element set, and B is a one-element set. 
Corollary 8.4 If $T$ is a taut functor, then the natural transformation $\mu$ is taut if diagram (2.4) is a (weak) pullback whenever A is empty and B is a one-element set.

Observation 8.5 (a) Let $A$ and $B$ be the empty and a one-element set, respectively. Then diagram (2.4) can be described as

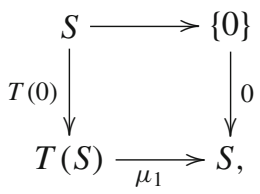

where $T(0)(s)(0)=s, T(0)(s)\left(s^{\prime}\right)=0$ for $s^{\prime} \neq 0$, and $\mu_{1}$ is defined by $\mu_{1}(t)=$ $\sum_{s \in S} t(s) s$.

(b) Let $A$ and $B$ be a two-element set and a one-element set, respectively (as in the proof of Theorem 7.1). Then diagram (2.4) can be described as

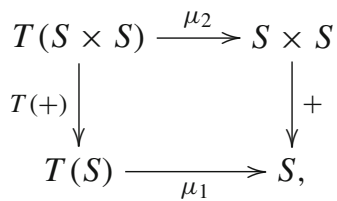

where $\mu_{1}$ is as above, and $T(+)$ and $\mu_{2}$ are defined by $T(+)(t)(s)=$ $\sum_{x+y=s} t(x, y)$ and

$$
\mu_{2}(t)=\left(\sum_{(x, y) \in S \times S} t(x, y) x, \sum_{(x, y) \in S \times S} t(x, y) y\right)
$$

respectively.

Lemma 8.6 If $A$ and $B$ are the empty and a one-element set, respectively, then diagram (2.4) is a weak pullback if and only if, for $s, s^{\prime} \in S$, we have:

(a) $s+s^{\prime}=0 \Rightarrow s=0=s^{\prime}$ (condition 6.1(iii));

(b) $s s^{\prime}=0 \Rightarrow\left(s=0 \vee s^{\prime}=0\right)$.

Proof As follows from Observation 8.5(a), when $A$ and $B$ are the empty and a oneelement set, respectively, diagram (2.4) is a weak pullback if and only if

$$
\forall t \in T(S)\left(\mu_{1}(t)=0 \Rightarrow \exists s \in S T(0)(s)=t\right) .
$$

Using the descriptions of $\mu_{1}$ and $T(0)$ in Observation 8.5(a), we can rewrite (8.7) as

$$
\forall t \in T(S)\left(\sum_{s \in S} t(s) s=0 \Rightarrow(s \neq 0 \Rightarrow t(s)=0)\right)
$$


After that we only need to prove that (8.8) is equivalent to the conjunction of conditions (a) and (b), which is straightforward:

Indeed, if (a) holds, then $\sum_{s \in S} t(s) s=0$ implies

$$
\forall s \in S \quad t(s) s=0
$$

which, by (b), implies $s \neq 0 \Rightarrow t(s)=0$. That is, (8.8) follows from the conjunction of conditions (a) and (b). On the other hand, if $s+s^{\prime}=0$, then $t$ in $T(S)$, defined by

$$
t(s)=1, t(1)=s^{\prime} \text {, and } t\left(s^{\prime \prime}\right)=0 \text { for } s \neq s^{\prime \prime} \neq 1 \text {, }
$$

has $\sum_{s \in S} t(s) s=1 s+s^{\prime} 1=s+s^{\prime}=0$, which, by (8.8), gives $s^{\prime}=0$, and then $s=s+s^{\prime}=0$. Therefore (8.8) implies (a). If $s s^{\prime}=0$, then $t \in T(S)$, defined by

$$
t\left(s^{\prime}\right)=s \text { and } t\left(s^{\prime \prime}\right)=0 \text { for } s^{\prime \prime} \neq s^{\prime},
$$

has $\sum_{s \in S} t(s) s=s s^{\prime}=0$, which, by (8.8), gives $s^{\prime} \neq 0 \Rightarrow s=0$.

Combining this lemma with Corollaries 6.5 and 8.4 we obtain the following characterization of taut free $S$-semimodule monads:

Theorem 8.7 The free $S$-semimodule monad $T$ is taut if and only if no non-zero element in $S$ has an additive inverse and no non-zero element of $S$ is a zero divisor.

Lemma 8.8 In the notation of (8.6), let

$$
\begin{aligned}
M(S) & =\left\{m \in T(S) \mid \forall a, b \in S\left(\mu_{1}(m)=a+b \Rightarrow \exists t \in T^{2}(S)(T(+)(t)\right.\right. \\
& \left.\left.\left.=m \& \mu_{2}(t)=(a, b)\right)\right)\right\}
\end{aligned}
$$

If $T$ is a weakly cartesian functor, then $M(S)$ is a submonoid in the additive monoid $T(S)$.

Proof If $m=0$ and $\mu_{1}(m)=a+b$, then $a=0=b$ by Lemma 6.1(iii). Therefore, for $m=0$ and $\mu_{1}(m)=a+b$, we have $T(+)(0)=m \& \mu_{2}(0)=(a, b)$. This implies that $M(S)$ contains 0 . For $m_{1}, m_{2} \in M(S)$ and $a, b \in S$ with $\mu_{1}\left(m_{1}+m_{2}\right)=a+b$, we observe:

- Since $\mu_{1}\left(m_{1}\right)+\mu_{1}\left(m_{2}\right)=a+b$, by Lemma 6.3 there exist $a_{1}, a_{2}, b_{1}, b_{2} \in S$ with

$$
\mu_{1}\left(m_{1}\right)=a_{1}+b_{1}, \mu_{1}\left(m_{2}\right)=a_{2}+b_{2}, a=a_{1}+a_{2}, b=b_{1}+b_{2} .
$$

- Then, since $m_{1}$ and $m_{2}$ are in $M(S)$, there exist $t_{1}$ and $t_{2}$ in $T^{2}(S)$ with

$$
T(+)\left(t_{1}\right)=m_{1}, \mu_{2}\left(t_{1}\right)=\left(a_{1}, b_{1}\right), T(+)\left(t_{2}\right)=m_{2}, \mu_{2}\left(t_{2}\right)=\left(a_{2}, b_{2}\right) .
$$

- Therefore $T(+)\left(t_{1}+t_{2}\right)=m_{1}+m_{2}$ and $\mu_{2}\left(t_{1}+t_{2}\right)=\left(a_{1}, b_{1}\right)+\left(a_{2}, b_{2}\right)=(a, b)$, which proves that $m_{1}+m_{2}$ is in $M(S)$. 
Lemma 8.9 Let $\mathbf{C}$ be any subset of $S$ that generates $S$ as an additive monoid. If $T$ is a weakly cartesian functor, then the following conditions are equivalent:

(i) diagram (2.4) is a weak pullback whenever $A$ is a two-element set, and B is a one-element set;

(ii) for every $a, b, c, d \in S$ with $c$ in $\mathbf{C}$ and $c d=a+b$, there exists a map

$$
t:\{(x, y) \in S \times S \mid x+y=d\} \longrightarrow S
$$

with

$$
\sum_{x+y=d} t(x, y) x=a, \sum_{x+y=d} t(x, y) y=b, \sum_{x+y=d} t(x, y)=c .
$$

Proof As follows from Observation 8.5(b), condition (i) holds if and only if diagram (8.6) is a weak pullback. Therefore condition (i) is equivalent to the equality $M(S)=$ $T(S)$, where $M(S)$ is defined as in Lemma 8.8. Let us write $[d, c]$ for the map $S \rightarrow S$ defined by $[d, c](d)=c$ and $s \neq d \Rightarrow[d, c](s)=0$. The set $\{[d, c] \mid d \in S, c \in C\}$ generates $T(S)$ as an additive monoid, and so the equality $M(S)=T(S)$ holds if and only if every $[d, c]$, with $d \in S$ and $c \in C$, is in $M(S)$. On the other hand, $[d, c]$ is in $M(S)$ if and only if, for every $a, b \in S$ with $c d=a+b$, there exists $t \in T^{2}(S)$ with

$$
\sum_{(x, y) \in S \times S} t(x, y) x=a, \quad \sum_{(x, y) \in S \times S} t(x, y) y=b, \quad \sum_{x+y=d} t(x, y)=c, \quad \sum_{x+y=S} t(x, y)=0,
$$

for all $s \neq d$. The fact that the existence of such a $t$ (for all $a, b, c, d \in S$ with $c$ in $C$ ) is equivalent to condition (ii) follows from Lemma 6.1(iii).

Now we are ready to present our main results on the free $S$-semimodule monad $T$ :

Theorem 8.10 The free $S$-semimodule monad $T$ satisfies $(B C)$ if and only if the semiring $S$ satisfies the following conditions:

(a) no non-zero element in $S$ has an additive inverse;

(b) for every $a, b, c, d \in S$ with $a+b=c+d$, there exist $x, y, z, t$ in $S$ with

$$
x+y=a, z+t=b, x+z=c, y+t=d ;
$$

(c) $S$ has no zero divisors, that is, $s s^{\prime}=0 \Rightarrow\left(s=0 \vee s^{\prime}=0\right)$ for $s, s^{\prime} \in S$;

(d) for every $a, b, c, d \in S$ with $c d=a+b$, there exists a map $t:\{(x, y) \in$ $S \times S \mid x+y=d\} \rightarrow S$ satisfying (8.11).

Furthermore, condition $(d)$ can be replaced by:

(e) there exists a subset $C$ of $S$ that generates $S$ as an additive monoid, and such that, for every $a, b, c, d \in S$ with $c$ in $C$ and $c d=a+b$, there exists a map $t:\{(x, y) \in S \times S \mid x+y=d\} \rightarrow S$ satisfying (8.11). 
Proof Note that conditions (a)-(e) coincide with conditions 6.1(iii), 6.3(iii), 8.6(b), 8.9(ii) with $C=S$, and 8.9(ii) respectively; for (e) $\Leftrightarrow 8.9$ (ii) note, however, that "any" can be understood as either existential or universal quantifier-which follows from Lemma 8.9. After that all we need is to recall that the functor $T$ satisfies (BC) if and only if $S$ satisfies 6.1(iii) and 6.3(iii) (Theorem 6.4), and to put together Corollary 8.3 and Lemmas 8.6 and 8.9.

From here and Theorem 7.1 we obtain:

Theorem 8.11 The free $S$-semimodule monad $T$ is weakly cartesian if and only if it satisfies $(B C)$ and the implication $s+s^{\prime}=1 \Rightarrow\left(s=0 \vee s^{\prime}=0\right)$ for $s, s^{\prime} \in S$.

\section{Semimodules IV: examples}

Conditions (b), (d), and (e) of Theorem 8.10 can be complicated to check, and we will use simple stronger conditions provided by

Proposition 9.1 (a) Iffor every $a, b \in S$ there exists $x \in S$ such that either $a+x=b$ or $b+x=a$, and the additive monoid of $S$ is a monoid with cancelation, then condition 8.10 (b) holds.

(b) If everynon-zero element in S has a multiplicative inverse and no non-zero element in $S$ has an additive inverse, then condition 8.10(d) holds.

(c) If $S$ is generated by $\{1\}$, then condition $8.10(\mathrm{e})$ holds.

Proof (a): Let $a, b, c, d \in S$ be as in Theorem 8.10(b). Without loss of generality we can assume that there exist $p, q, r \in S$ with $a+p=b, a+q=c, a+r=d$, after which we can take $x=0, y=a, z=c$, and $t=r$. Indeed, we will obtain $x+y=0+a=a, z+t=c+r=b$ since $a+c+r=c+d=a+b$, $x+z=0+c=c$, and $y+t=a+r=d$.

(b): Given $a, b, c, d \in S$ with $c d=a+b$, consider the two cases $d=0$ and $d \neq 0$ separately. If $d=0$, then $a=0=b$, and

$$
t:\{(x, y) \in S \times S \mid x+y=d\}=\{(0,0)\} \longrightarrow S
$$

defined by $t(0,0)=c$ satisfies (8.11). If $d \neq 0$, then $t:\{(x, y) \in S \times S \mid x+y=$ $d\} \rightarrow S$, defined by

$$
t(d, 0)=a d^{-1}, t(0, d)=b d^{-1},(d, 0) \neq(x, y) \neq(0, d) \Rightarrow t(x, y)=0,
$$

also satisfies (8.11).

(c): We take $C=\{1\}$ and then to take $a, b, c, d \in S$ with $c$ in $C$ and $c d=a+b$ is to take $a, b, d \in S$ with $d=a+b$. Then $t:\{(x, y) \in S \times S \mid x+y=d\} \rightarrow S$, defined by

$$
t(a, b)=1 \text { and }(x, y) \neq(a, b) \Rightarrow t(x, y)=0
$$

satisfies (8.11). 
Example 9.2 If $S=\mathbb{N}$ is the semiring of natural numbers, then all our conditions are satisfied. In particular for conditions 8.10(b) and 8.10(e) this follows from Propositions 9.1(a) and 9.1(c) respectively. In this case $S$-semimodules are nothing but commutative monoids, and we conclude that the free commutative monoid monad is weakly cartesian. Note that it is not cartesian by Proposition 5.1.

Example 9.3 Let $S=\{0,1\}$ be the quotient semiring of $\mathbb{N}$ determined by $1+1=1$. Then the $S$-semimodules are nothing but commutative idempotent monoids, and so, as trivially follows from Theorem 8.11, the free commutative idempotent monoid monad is not weakly cartesian. However, it satisfies (BC), since $S=\{0,1\}$ satisfies all conditions of Theorem 8.10. For condition 8.10(e) it follows again from Proposition 9.1(c), while condition 8.10(b) can be easily checked directly. Note that Proposition 9.1(a) cannot be used here since the additive monoid of $S$ does not admit cancelation. The fact that the free commutative idempotent monoid monad satisfies (BC) is in fact known and was already mentioned e.g. in [8].

Example 9.4 In spite of Examples 9.2 and 9.3, the "intermediate" semiring $S=$ $\{0,1,2\}$, having $2+1=2$, does not satisfy condition 8.10 (b), and so in this case the free $S$-semimodule functor does not even preserve weak pullbacks. Indeed, for $a=b=1$ and $c=d=2$, we have $a+b=c+d$, but there is no $x, y, z, t$ in $S$ with

$$
x+y=a, z+t=b, x+z=c, y+t=d .
$$

This shows the cancelation requirement cannot be removed in Proposition 9.1(a), as Example 9.3 might seem to suggest.

Example 9.5 If $S=\mathbb{Q}_{+}$is the semiring of non-negative rational numbers, then, as in Example 9.3, the free $S$-semimodule monad is not weakly cartesian (again, trivially from Theorem 8.11) but satisfies (BC). However, Proposition 8.10(c) cannot be used here, and Proposition 9.1(b) should be used instead, which makes condition 8.10(d) trivial. The same is true if $S=\mathbb{R}_{+}$is the semiring of non-negative real numbers.

Example 9.6 If the semirings $S$ and $S^{\prime}$ satisfy conditions 8.10(a) and 8.10(b), then the product $S \times S^{\prime}$ also does. But it never satisfies condition 8.10(c) unless one of the two rings is trivial. This gives free semimodule monads which do not satisfy (BC) although the functor $T$ does.

Acknowledgments We are grateful to Lurdes Sousa, who showed us that Theorem 6.4 is essentially the same as Theorem 5.13 in the paper [10] of Gumm and Schröder. We are also grateful to the referee for mentioning to us the relationship between regularity and tautness.

\section{References}

1. Bourn, D.: Normalization equivalence, kernel equivalence and affine categories. Lecture Notes Math. 1488, 43-62 (1991)

2. Carboni, A., Johnstone, P.T.: Connected limits, familial representability and Artin glueing. Math. Struct. Comput. Sci. 5, 441-459 (1995)

3. Carboni, A., Johnstone, P.T.: Corrigenda for "Connected limits, familial representability and Artin glueing”. Math. Struct. Comput. Sci. 14, 185-187 (2004) 
4. Clementino, M.M., Hofmann, D.: Topological features of lax algebras. Appl. Categ. Struct. 11, 267$286(2003)$

5. Clementino, M.M., Hofmann, D.: On extensions of lax monads. Theory Appl. Categ. 13, 41-60 (2004)

6. Clementino, M.M., Hofmann, D.: Lawvere completeness in topology. Appl. Categ. Struct. 17, 175-210 (2009)

7. Clementino, M.M., Hofmann, D.: Descent morphisms and a van Kampen Theorem in categories of lax algebras. Topology Appl. 159, 2310-2319 (2012)

8. Clementino, M.M., Hofmann, D., Janelidze, G.: On exponentiability of étale algebraic homomorphisms. J. Pure Appl. Algebra 217, 1195-1207 (2013)

9. Csákány, B.: Primitive classes of algebras which are equivalent to classes of semi-modules and modules. Acta Sci. Math. (Szeged) 24, 157-164 (1963)

10. Gumm, H.P., Schröder, T.: Monoid-labeled transition systems. Electr. Notes Theor. Comput. Sci. 44(1) (2001)

11. Gumm, H.P., Ursini, A.: Ideals in universal algebras. Algebra Universalis 19, 45-54 (1984)

12. Higgins, P.J.: Groups with multiple operators. Proc. Lond. Math. Soc. 6(3), 366-416 (1956)

13. Janelidze, G., Márki, L., Tholen, W.: Semi-abelian categories. J. Pure Appl. Algebra 168, 367-386 (2002)

14. Janelidze, G., Márki, L., Tholen, W., Ursini, A.: Ideal determined categories. Cah. Topol. Géom. Différ. Catég. 51(2), 115-125 (2010)

15. Janelidze, G., Márki, L., Ursini, A.: Ideals and clots in universal algebra and in semi-abelian categories. J. Algebra 307(1), 191-208 (2007)

16. Janelidze, Z.: The pointed subobject functor, $3 \times 3$ lemmas, and subtractivity of spans. Theory Appl. Categ. 23(11), 221-242 (2010)

17. Johnstone, P.T.: A note on the semiabelian variety of Heyting semilattices. Fields Inst. Commun. 43, 317-318 (2004)

18. Koslowski, J.: A monadic approach to policategories. Theory Appl. Categ. 14(7), 125-156 (2005)

19. Leinster, T.: Higher Operads, Higher Categories, London Mathematical Society Lecture Note Series 298. Cambridge University Press, Cambridge (2004)

20. Manes, E.G.: Implementing collection classes with monads. Math. Struct. Comput. Sci. 8, 231-276 (1998)

21. Manes, E.G.: Taut monads and T0-spaces. Theor. Comput. Sci. 275, 79-109 (2002)

22. Möbus, A.: Alexandrov compactification of relational algebras. Arch. Math. 40, 526-537 (1983)

23. Szawiel, S., Zawadowski, M.: Theories of analytic monads (2012, preprint, arxiv 1204.2703)

24. Szawiel, S., Zawadowski, M.: Monads of regular theories Appl. Categ. Struct. (2012). doi:10.1007/ s10485-013-9331-x

25. Ursini, A.: Sulle varietà di algebre con una buona teoria degli ideali. Boll. Un. Mat. Ital. 6(4), 90-95 (1972)

26. Ursini, A.: On subtractive varieties I. Algebra Universalis 31, 204-222 (1994)

27. Weber, M.: Generic morphisms, parametric representations and weakly cartesian monads. Theory Appl. Categ. 13, 191-234 (2004) 